\title{
Pooled analysis of genome-wide association studies of cervical intraepithelial neoplasia 3 (CIN3) identifies a new susceptibility locus
}

\author{
Dan Chen ${ }^{1,2}$, Stefan Enroth ${ }^{2}$, Han Liu ${ }^{1}$, Yang Sun $^{3}$, Huibo Wang ${ }^{4}$, Min Yu ${ }^{3}$, Lian \\ Deng $^{5,6}$, Shuhua $X \mathbf{u}^{5,6,7,8}$, Ulf Gyllensten ${ }^{2}$ \\ ${ }^{1}$ Ministry of Education and Shanghai Key Laboratory of Children's Environmental Health, Xin Hua Hospital Affiliated to \\ Shanghai Jiao Tong University School of Medicine, Shanghai, China \\ ${ }^{2}$ Department of Immunology, Genetics and Pathology, Science for Life Laboratory Uppsala, Uppsala University, Uppsala, \\ Sweden \\ ${ }^{3}$ Laboratory of Biochemistry and Molecular Biology, School of Life Science, Yunnan University, Kunming, China \\ ${ }^{4}$ Department of Neurosurgery, First Affiliated Hospital of Nanjing Medical University, Nanjing, China \\ ${ }^{5}$ Chinese Academy of Sciences (CAS) Key Laboratory of Computational Biology, Max Planck Independent Research Group \\ on Population Genomics, CAS-MPG Partner Institute for Computational Biology (PICB), Shanghai Institutes for Biological \\ Sciences, CAS, Shanghai, China \\ ${ }^{6}$ University of Chinese Academy of Sciences, Beijing, China \\ ${ }^{7}$ School of Life Science and Technology, Shanghai Tech University, Shanghai, China \\ ${ }^{8}$ Collaborative Innovation Center of Genetics and Development, Shanghai, China
}

Correspondence to: Dan Chen, email: simpledandan1981@163.com, simpledandan@shsmu.edu.cn

Keywords: cervical intraepithelial neoplasia 3, genome-wide association study, genetic variants, expression quantitative trait locus, human leukocyte antigen

Received: December 15, $2015 \quad$ Accepted: May 13, $2016 \quad$ Published: June 07, 2016

\section{ABSTRACT}

Recent genome-wide association studies (GWASs) in subjects of European descent have identified associations between cervical cancer risk and three independent loci as well as multiple classical human leukocyte antigen (HLA) alleles at 6p21.3. To search for novel loci associated with development of cervical cancer, we performed a pooled analysis of data from two GWASs by imputing over 10 million genetic variants and 424 classical HLA alleles, for 1,553 intraepithelial neoplasia 3 (CIN3), 81 cervical cancer and 4,442 controls from the Swedish population. Notable findings were validated in an independent study of 961 patients ( 827 with CIN3 and 123 with cervical cancer) and 1,725 controls. Our data provided increased support for previously identified loci at 6p21.3 (rs9271898, $P=1.2 \times 10^{-24}$; rs2516448, $1.1 \times 10^{-15}$; and rs3130196, $2.3 \times 10^{-9}$, respectively) and also confirmed associations with reported classical HLA alleles including $H L A-B * 07: 02,-B * 15: 01,-D R B 1 * 13: 01,-D R B 1 * 15: 01,-D Q A 1 * 01: 03,-D Q B 1 * 06: 03$ and $-D Q B 1 * 06: 02$. In addition, we identified and subsequently replicated an independent signal at rs73730372 at $6 \mathrm{p} 21.3$ (odds ratio $=0.60,95 \%$ confidence interval $=0.54-0.67$, $P=3.0 \times 10^{-19}$ ), which was found to be an expression quantitative trait locus (eQTL) of both HLA-DQA1 and HLA-DQB1. This is one of the strongest common genetic protective variants identified so far for CIN3. We also found $H L A-C * 07: 02$ to be associated with risk of CIN3. The present study provides new insights into pathogenesis of CIN3.

\section{INTRODUCTION}

Worldwide, cervical cancer is the fourth most common cancer in women [1]. Persistent infection with carcinogenic human papillomavirus (HPV) strains is the main cause of cervical cancer and its precursor lesions, cervical intraepithelial neoplasia (CIN) [2]. More advanced lesions, designated CIN3, are considered to be the same as carcinoma in situ (CIS) or Stage 0 cervical cancer. Although both population screening and a prophylactic vaccine are available, cervical cancer continues to be a major threat to female health globally. 
Therefore, further understanding of the contribution of genetic susceptibility to persistent HPV infection as well as tumorigenesis is important for preventing the disease.

We recently performed the first genome-wide association study (GWAS) of cervical precancer (CIN3) in a Swedish population and confirmed previously reported associations with classical human leukocyte antigen (HLA) alleles, including $H L A-B^{*} 07: 02,-D R B 1 * 13: 01$, $-D R B 1 * 15: 01, \quad-D Q A 1 * 01: 03, \quad-D Q B 1 * 06: 03$ and $-D Q B 1 * 06: 02$. In addition, we identified three novel loci in the major histocompatibility complex (MHC) region at 6p21.3, rs9272143 between HLA-DRB1 and HLA-DQA1, rs2516448 adjacent to the MHC class I polypeptide-related sequence A gene (MICA) and rs3117027 at of HLA-DPB2, which acted independently of classical HLA alleles [3].

The statistical power of individual GWAS has been limited by the modest effect size of genetic variants and the number of variants that could be studied. To identify additional cervical precancer susceptibility loci, we expanded the present GWAS by pooling its data with the data from another GWAS, together providing data on 1,553 cases with CIN3, 81 cases with cervical cancer and 4,442 controls, all from the Swedish population. To bring genotype data obtained from different single-nucleotide polymorphism (SNP) arrays into a common framework and provide information on ungenotyped genetic variants, we imputed $>10$ million genetic variants and 424 classical HLA alleles, using the 1000 Genome Project data and the Type 1 Diabetes Genetics Consortium (T1DGC) panel as reference. We then conducted a comprehensive examination of the association between CIN3 risk and common variants, rare variants as well as classical HLA alleles. Notable findings were validated in an independent study of 961 cases ( 827 with CIN3 and 123 with cervical cancer) and 1,725 controls.

\section{RESULTS}

\section{Genome-wide association results}

After stringent quality control (QC), data from 1,634 cases (1,553 CIN3 and 81 cervical cancer) and 4,442 controls were available for 5,471,179 SNPs with an overall call rate of $99.32 \%$. The pooled analysis showed modest evidence of over-dispersion $(\lambda=1.08)$. Genetic variants mapping to the previously identified susceptiblity loci at $6 \mathrm{p} 21.3$ provided the best evidence for an association with cervical precancer (Supplementary Figure S1). A total of 1,576 SNPs showed evidence for an association with CIN3 risk at $P=5 \times 10^{-7}$, 1,575 of which are located within the $\mathrm{MHC}$ region at $6 \mathrm{p} 21.3$ and one of which is located at 18q12.3. After excluding the genetic variants in the extended MHC region where extensive LD extends over long distances, $\lambda$ is only 1.02 (Supplementary Figure S1). The strongest association was attained for rs 9271898 (odds ratio $[\mathrm{OR}]=0.64,95 \%$ confidence interval $[\mathrm{CI}]=0.59-0.70, P=1.2 \times 10^{-24}$ for the minor allele A), which is located between $H L A-D R B 1$ and $H L A-D Q A 1$ and is highly correlated with rs9272143 $\left(D^{\prime}=0.99, r^{2}=0.98\right)$, the top hit previously reported (Table 1). A borderline genome-wide significant association was observed between CIN3 risk and a locus outside MHC region, that is $\mathrm{rs} 73000875$ at $18 \mathrm{q} 12.3(\mathrm{OR}=0.61,95 \%$ $\mathrm{CI}=0.50-0.74, P=3.8 \times 10^{-7}$ for the minor allele $\left.\mathrm{G}\right)$.

Concordance between the genotyped and imputed data was 0.96 for $H L A-A, 0.97$ for $H L A-B, 0.92$ for $H L A$ $D R B 1,0.92$ for $H L A-D Q B 1$ and 0.91 for $H L A-D P B 1$, at two field level of HLA typing, respectively. As shown in Table 2, three HLA class I alleles showed evidence of association with CIN3 at the $5 \times 10^{-7}$ threshold, namely $H L A-B * 07: 02(\mathrm{OR}=1.41,95 \% \mathrm{CI}=1.27-1.57, P=1.4$ $\left.\times 10^{-10}\right), H L A-B^{*} 15: 01(\mathrm{OR}=0.67,95 \% \mathrm{CI}=0.58-0.77$, $\left.P=2.6 \times 10^{-8}\right)$ and HLA-C*07:02 (OR $=1.37,95 \%$ $\left.\mathrm{CI}=1.24-1.52, P=2.6 \times 10^{-9}\right) . H L A-B^{*} 07: 02$ and $H L A-C * 07: 02$ are in strong LD with each other $\left(r^{2}=0.87\right.$, $D^{\prime}=0.96$ in controls). Five HLA class II alleles were associated with risk of CIN3 namely $H L A-D R B 1 * 13: 01$ $\left(\mathrm{OR}=0.49,95 \% \mathrm{CI}=0.40-0.59, P=1.8 \times 10^{-13}\right), H L A-$ $D R B 1 * 15: 01(\mathrm{OR}=1.36,95 \% \mathrm{CI}=1.22-1.51, P=$ $\left.9.7 \times 10^{-9}\right), H L A-D Q A 1 * 01: 03(\mathrm{OR}=0.49,95 \% \mathrm{CI}=$ $\left.0.40-0.59, P=5.6 \times 10^{-14}\right), H L A-D Q B 1 * 06: 03(\mathrm{OR}=$ $\left.0.54,95 \% \mathrm{CI}=0.45-0.64, P=1.5 \times 10^{-11}\right)$, and HLA$D Q B 1 * 06: 02(\mathrm{OR}=1.32,95 \% \mathrm{CI}=1.19-1.47, P=2.5$ $\left.\times 10^{-7}\right) . H L A-D R B 1 * 15: 01$ and HLA-DQB1*06:02 $\left(r^{2}=\right.$ $0.95, D^{\prime}=0.99$ in controls), as well as $H L A-D R B 1 * 13: 01$, $H L A-D Q A 1 * 01: 03$ and $H L A-D Q B 1 * 06: 03$ are in strong LD with each other $\left(r^{2}=0.96\right.$ and $D^{\prime}=0.99$ between $D R B 1^{*} 13: 01$ and $D Q A 1^{*} 01: 03 ; r^{2}=0.93, D^{\prime}=0.99$ between $D R B 1 * 13: 01$ and $D Q B 1 * 06: 03 ; r^{2}=0.91, D^{\prime}=$ 0.96 between $D Q A 1 * 01: 03$ and $D Q B 1 * 06: 03$ in controls), respectively (Supplementary Table $\mathrm{S} 1$ ).

To evaluate the independence of associations at $6 \mathrm{p} 21.3$, we conducted stepwise logistic regression analysis at 6p21.3 (Figure 1, Table 1, Supplementary Figure S2). Consistent with previous findings, after conditioning on the top signal rs9271898, the strongest secondary signal appeared to be for rs2516448 (OR $=1.41,95 \% \mathrm{CI}=1.30-1.54, P=5.6 \times 10^{-16}$ compared with $\mathrm{OR}=1.39,95 \% \mathrm{CI}=1.28-1.52, P=1.1 \times 10^{-15}$ for the minor allele $\mathrm{T}$ for the unconditional analysis), which is located downstream of $M I C A$. When further conditioning upon rs2516448, residual association was detected at some SNPs and HLA alleles in this region, with the most significant one occurring at rs3130196 (OR $=1.40,95 \% \mathrm{CI}=1.25-1.57, P=9.4 \times 10^{-9}$ compared with $\mathrm{OR}=1.40,95 \% \mathrm{CI}=1.26-1.57, P=2.3 \times 10^{-9}$ for the minor allele $\mathrm{C}$ for the unconditional analysis), which is in LD with $\operatorname{rs} 3117027\left(D^{\prime}=0.96, r^{2}=0.32\right)$ and is located downstream of $H L A-D P B 1$ and upstream of $H L A-D P A 1$. Conditioning on all three SNPs jointly still left residual association at rs $115625939(\mathrm{OR}=0.68,95 \% \mathrm{CI}=0.58$ $0.79, P=3.2 \times 10^{-7}$ compared with $\mathrm{OR}=0.58,95 \% \mathrm{CI}=$ $0.51-0.67, P=1.4 \times 10^{-15}$ for the minor allele $\mathrm{G}$ for the 
Table 1: Summary of genome-wide association study results of 4 SNPs at 6 p21.3 independently associated with CIN3

\begin{tabular}{|c|c|c|c|c|c|c|c|c|c|c|}
\hline \multirow[b]{2}{*}{ Loci } & \multirow[b]{2}{*}{ SNP } & \multirow[b]{2}{*}{ Position $^{\mathrm{a}}$} & \multirow[b]{2}{*}{ Nearby gene } & \multirow[b]{2}{*}{ Alleles $^{b}$} & \multicolumn{2}{|c|}{ MAF } & \multicolumn{2}{|c|}{ Single SNP ${ }^{c}$} & \multicolumn{2}{|c|}{ Stepwise conditional analysis } \\
\hline & & & & & Case & Control & OR $(95 \% C I)$ & $\boldsymbol{P}$ & OR $(95 \% C I)$ & $\boldsymbol{P}$ \\
\hline 1 & rs9271898 & $\begin{array}{c}32595972 \\
\text { (class I) }\end{array}$ & $\begin{array}{l}H L A-D R B 1, \\
H L A-D Q A 1\end{array}$ & $\mathrm{G}>\mathrm{A}$ & 0.38 & 0.49 & $0.64(0.59-0.70)$ & $1.2 \times 10^{-24}$ & - & - \\
\hline 2 & rs2516448 & $\begin{array}{c}31390410 \\
(\text { class I) }\end{array}$ & $M I C A$ & $\mathrm{C}>\mathrm{T}$ & 0.58 & 0.50 & $1.39(1.28-1.52)$ & $1.1 \times 10^{-15}$ & $1.41(1.30-1.54)^{\mathrm{d}}$ & $5.6 \times 10^{-16 \mathrm{~d}}$ \\
\hline 3 & rs3130196 & $\begin{array}{c}33063219 \\
\text { (class II) }\end{array}$ & $\begin{array}{c}H L A-D P B 1 \\
H L A-D P A 1\end{array}$ & $\mathrm{~T}>\mathrm{C}$ & 0.17 & 0.13 & $1.40(1.26-1.57)$ & $2.3 \times 10^{-9}$ & $1.40(1.25-1.57)^{\mathrm{e}}$ & $9.4 \times 10^{-9} \mathrm{e}$ \\
\hline 4 & rs 115625939 & $\begin{array}{c}32583584 \\
\text { (class II) }\end{array}$ & $\begin{array}{l}H L A-D R B 1 \\
H L A-D Q A 1\end{array}$ & $\mathrm{~A}>\mathrm{G}$ & 0.09 & 0.15 & $0.58(0.51-0.67)$ & $1.4 \times 10^{-15}$ & $0.68(0.58-0.79)^{\mathrm{f}}$ & $3.2 \times 10^{-7 \mathrm{f}}$ \\
\hline
\end{tabular}

MAF, minor allele frequency; OR, odds ratio; CI, confidence interval; $P$, two-sided $P$ value corresponding to the OR.

${ }^{\mathrm{a}} \mathrm{GRCh} 37 / \mathrm{hg} 19$ Assembly.

${ }^{b}$ Major allele > Minor allele

${ }^{\mathrm{c}} \mathrm{ORs}$ and $95 \%$ CIs for minor allele in log-additive model were derived from logistic regression with adjustment for study and one significant principal component generated by principal components analysis.

${ }^{\mathrm{d} C}$ Conditioning on rs 9271898.

${ }^{\mathrm{e} C}$ Conditioning on rs 9271898 and rs 2516448

${ }^{\mathrm{f} C o n d i t i o n i n g ~ o n ~ r s 9271898, ~ r s 2516448 ~ a n d ~ r s 3130196 . ~}$
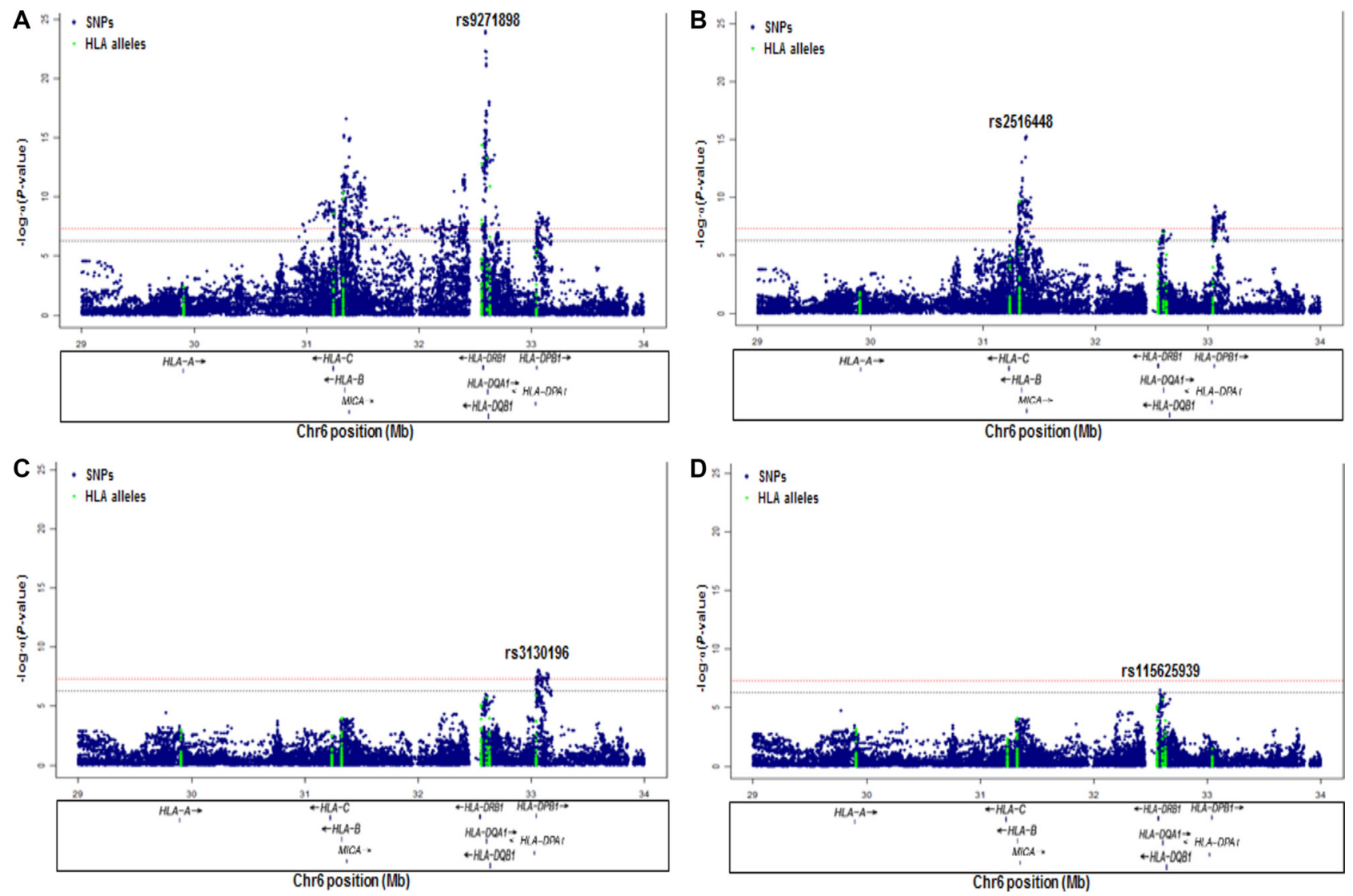

Figure 1: Association results of stepwise conditional logistic regression analysis for CIN3 at 6 p21.3. The - $\log 10(P$ values $)$ for each SNP (y-axis) are plotted against their position (x-axis) on chromosome 6 (hg19). The horizontal red line and blue line represent $P=5 \times 10^{-8}$ and $P=5 \times 10^{-7}$, respectively. (A) Association results in unconditional logistic regression analysis. (B) Association results upon conditioning on rs9271898. (C) Association results upon conditioning on rs9271898 and rs2516448. (D) Association results upon conditioning on rs9271898, rs2516448 and rs3130196. 
Table 2: Analysis of possible confounding of the classical HLA allele associations by SNPs

\begin{tabular}{|c|c|c|c|c|c|c|}
\hline & \multicolumn{2}{|c|}{ Allele frequency } & \multicolumn{2}{|c|}{ Original results $^{\mathrm{a}}$} & \multicolumn{2}{|c|}{ Conditioning on $4 \mathrm{SNPs}^{\mathrm{b}}$} \\
\hline & Case & Control & OR $(95 \%)$ & $P$ & OR $(95 \%)$ & $P$ \\
\hline \multicolumn{7}{|l|}{ HLA-B } \\
\hline$H L A \_B * 07: 02$ & 0.20 & 0.15 & $1.41(1.27-1.57)$ & $1.4 \times 10^{-10}$ & $1.08(0.96-1.22)$ & 0.20 \\
\hline$H L A \_B * 15: 01$ & 0.08 & 0.12 & $0.67(0.58-0.77)$ & $2.6 \times 10^{-8}$ & $0.76(0.65-0.89)$ & $5.1 \times 10^{-4}$ \\
\hline \multicolumn{7}{|l|}{ HLA-C } \\
\hline$H L A \_C^{*} 07: 02$ & 0.20 & 0.16 & $1.37(1.24-1.52)$ & $2.6 \times 10^{-9}$ & $1.06(0.94-1.19)$ & 0.38 \\
\hline \multicolumn{7}{|l|}{ HLA-DRB1 } \\
\hline$H L A \_D R B 1 * 13: 01$ & 0.04 & 0.08 & $0.49(0.40-0.59)$ & $1.8 \times 10^{-13}$ & $0.81(0.62-1.04)$ & 0.10 \\
\hline$H L A \_D R B 1 * 15: 01$ & 0.20 & 0.15 & $1.36(1.22-1.51)$ & $9.7 \times 10^{-9}$ & $1.01(0.90-1.14)$ & 0.84 \\
\hline \multicolumn{7}{|l|}{ HLA-DQA1 } \\
\hline$H L A \_D Q A 1 * 01: 03$ & 0.04 & 0.08 & $0.49(0.40-0.59)$ & $5.6 \times 10^{-14}$ & $0.76(0.59-0.98)$ & 0.03 \\
\hline \multicolumn{7}{|l|}{ HLA-DQB1 } \\
\hline$H L A \_D Q B 1^{*} 06: 03$ & 0.05 & 0.08 & $0.54(0.45-0.64)$ & $1.5 \times 10^{-11}$ & $0.89(0.70-1.13)$ & 0.35 \\
\hline$H L A \_D Q B 1 * 06: 02$ & 0.19 & 0.15 & $1.32(1.19-1.47)$ & $2.5 \times 10^{-7}$ & $0.98(0.86-1.10)$ & 0.69 \\
\hline
\end{tabular}

CI, confidence interval; HLA, human leukocyte antigen; SNP, single-nucleotide polymorphism; OR, odds ratio; P, two-sided $P$ value corresponding to the $O R$.

${ }^{\text {a }}$ Derived from logistic regression in log-additive model with adjustment for study and one informative eigenvector generated by principal components analysis.

${ }^{\mathrm{b}}$ Derived from logistic regression in log-additive model conditioning on rs9271898, rs2516448, rs3130196 and rs73730372 with adjustment for study and one significant principal component generated by principal components analysis.

unconditional analysis (Figure 2)). Only $H L A-B^{*} 15: 01$ showed statistically significant association after further conditioning on $\mathrm{rs} 115625939(\mathrm{OR}=0.76,95 \% \mathrm{CI}=$ $\left.0.65-0.89, P=5.1 \times 10^{-4}\right)$ (Table 2). The LD between rs 115625939 and the other three SNPs is weak $\left(r^{2}=0.16\right.$ with rs9271898; $r^{2}=0$ with $\operatorname{rs} 2516448$ and $r^{2}=0.01$ with rs3130196, respectively) (Supplementary Table S1). The LD between the significant SNPs and classical HLA alleles is shown in Supplementary Table S1. Statistially significant associations were observed for rs 9271898 (OR $\left.=0.67,95 \% \mathrm{CI}=0.61-0.74, P=1.0 \times 10^{-15}\right), \mathrm{rs} 2516448$ $\left(\mathrm{OR}=1.25,95 \% \mathrm{CI}=1.14-1.37, P=6.1 \times 10^{-6}\right)$, rs3130196 $(\mathrm{OR}=1.42,95 \% \mathrm{CI}=1.27-1.59, P=1.0$ $\left.\times 10^{-9}\right)$ and $\mathrm{rs} 115625939(\mathrm{OR}=0.71,95 \% \mathrm{CI}=0.60-0.85$, $\left.P=1.9 \times 10^{-4}\right)$ upon conditioning on significant HLA alleles/haplotypes, respectively (Supplementary Table S2). Collectively, these data provide evidence for a novel disease locus annotated by rs115625939 at $6 \mathrm{p} 21.3$, in addition to the three previously reported susceptibility loci in this region. The genetic variants that were highly correlated with each of the four independent loci $\left(r^{2} \geq 0.8\right)$ were grouped in bins and are shown in Supplementary Table S3. Similar associations were observed for the top signals when analyzing the CIN3 as a distinct group (Supplementary Table S4).

\section{Replication and combined results}

A genotyping assay for replication of rs 115625939 at 6 p21.3 was not possible to design. Instead, a strong proxy of $\operatorname{rs} 115625939, \operatorname{rs} 73730372\left(D^{\prime}=1, r^{2}=1\right)$, was successfully genotyped in the replication study, including an independent set of 956 cases (827 with CIN3 and 123 with cervical cancer) and 1,715 control subjects. The association between rs73730372 and CIN3 risk was independently replicated in the replication study $\left(\mathrm{OR}=0.64,95 \% \mathrm{CI}=0.54-0.77, P=9.8 \times 10^{-7}\right.$ for the minor allele A) with no evidence of heterogeneity between two study phases $(P$-heterogeneity $=0.39)$. In contrast, no association was observed between rs73000875 at $18 \mathrm{q} 12.3(\mathrm{OR}=0.88,95 \% \mathrm{CI}=0.69-1.11$, $P=0.26$ for the minor allele $\mathrm{G}$ ) and risk of CIN3 in the replication series.

After pooling the discovery and replication data, the ORs $(95 \% \mathrm{CI})$ were $0.62(0.55-0.70)$ and $0.31(0.19-0.51)$ for heterozygous (genotype CT) and homozygous carriers (genotype TT) of rs 73730372, compared to CC, respectively, yielding $P=3.0 \times 10^{-19}$ for trend in the combined analysis (Figure 3). No heterogeneity for the association of rs 73730372 was noted by tumor grade (CIN3 vs cervical cancer). 


\section{DISCUSSION}

We performed a pooled analysis of data from two GWASs by imputing over 10 million genetic variants and 424 classic HLA alleles. The results provided increased support for multiple independent susceptibility loci and classic HLA alleles within the MHC region, previously identified to be associated with cervical cancer. We have also provided support for the existence of a novel, independent, disease locus, as indicated by the SNP rs73730372 at $6 \mathrm{p} 21.3$. These findings suggest that immunological factors play an important role in the pathogenesis of CIN3.The risk or protective alleles may affect the binding affinity to high-risk HPVs or expression level of HLA molecules, hence influence susceptibility to infection and/or persistence of high-risk HPVs.

The minor allele of rs 73730372 was associated with a $40 \%$ reduced risk of CIN3 with the homozygous genotype conferring a $69 \%$ reduced risk (Figure 2). This is one of the strongest common genetic protective variants identified CIN3 SNP rs 73730372 correlates $\left(r^{2} \geq\right.$ $0.9)$ with 16 SNPs. It is located $11 \mathrm{~kb}$ upstream of $H L A$ -
$D Q A 1$ and $45 \mathrm{~kb}$ downstream of $H L A-D Q B 1$. rs 73730372 has recently been identified as expression quantitative trait locus (eQTL) that influence the expression of both HLA-DQA1 and HLA-DQB1 in blood [4]. HLA-DQA1 and $H L A-D Q B I$ belong to the HLA class II alpha and beta chain paralogs, respectively. Together, their gene products form a protein complex called an antigen-binding DQ $\alpha \beta$ heterodimer. This complex presents foreign peptides to the immune system to trigger the body's immune response, and therefore play a key role in the immune recognition process and subsequent clearance of virally-infected cells. Class II molecules are expressed in antigen presenting cells (APC: B Lymphocytes, dendritic cells, macrophages) and are known to be important in the regulation of the immune response to viral and other infections. Class II molecules present antigenic peptides to $\mathrm{CD}^{+}{ }^{+} \mathrm{T}$ helper cells to initiate a cell-mediated immune response [5]. Our study points to the importance of expression level of $H L A-D Q A 1$ and $H L A-D Q B 1$ for the susceptibility to develop cervical carcinoma. Impaired class II gene expression has been reported in genital HPV infections and in lesions caused by HPV [6-8]. The increased incidence and progression

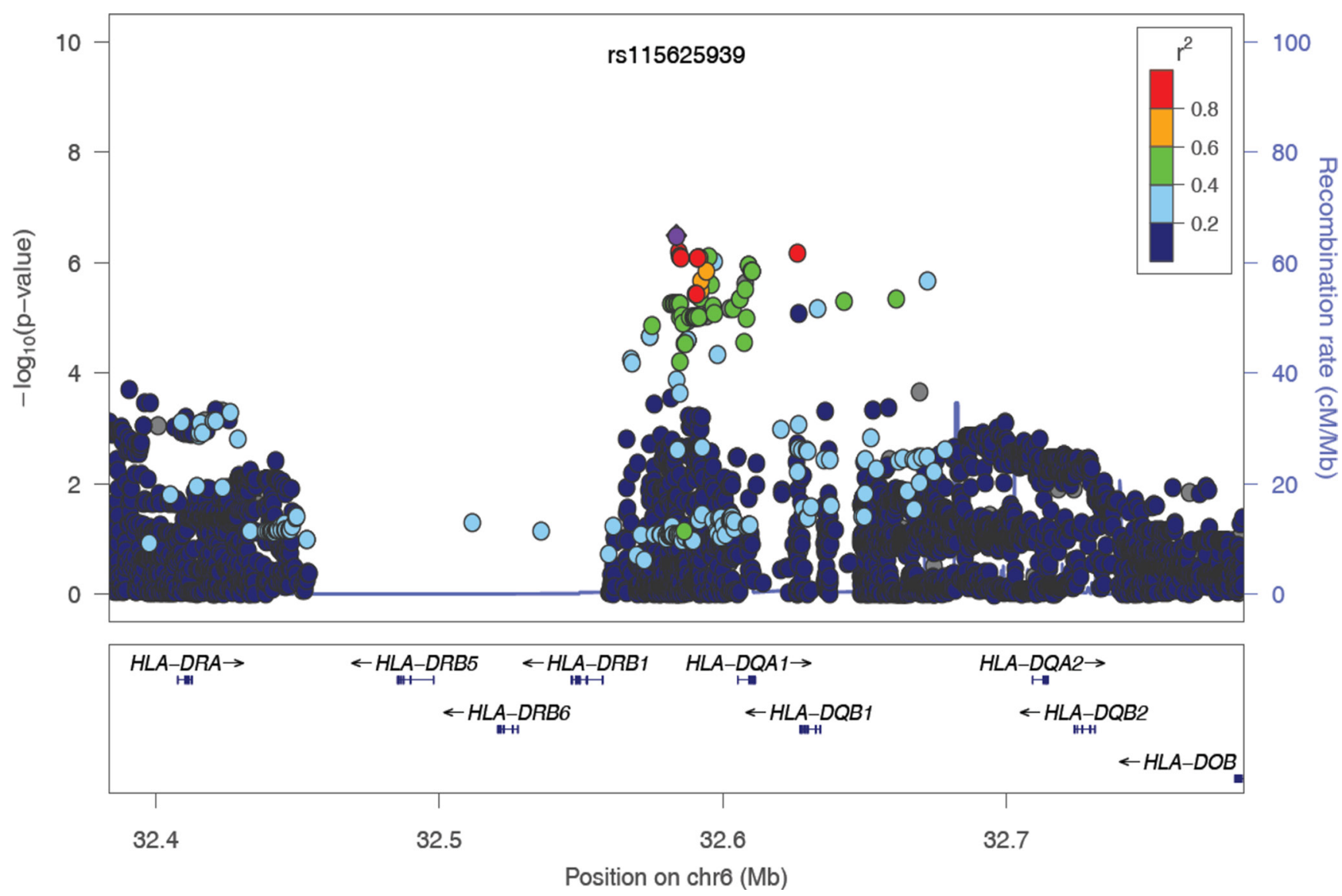

Figure 2: The regional association plot of rs115625939 in the MHC region. Results are shown for SNPs in the region flanking $200 \mathrm{~kb}$ on either side of rs 115625939 upon conditioning on the top 3 hits. The purple circle indicates the top SNP in each locus and the color of the dots represents the degree of linkage disequilibrium (based on $r^{2}$ ) in relation to the top SNP. Recombination rates (cM/Mb) overlay the plots and are based on HapMap data http://hapmap.ncbi.nlm.nih.gov/downloads/recombination/2008-03_rel22_B36/rates/. Genes in the region are represented with arrow heads indicating the direction of transcription. 
of HPV infections in immunosuppressed individuals illustrates the critical importance of the $\mathrm{CD}^{+}{ }^{+} \mathrm{T}$-cellregulated cell-mediated immune response in the resolution of HPV infection [5, 9]. Failure to develop an effective cell-mediated immune response to clear infection results in a persistent infection and, in the case of the oncogenic HPVs, an increased probability of progression to CIN3 and cervical cancer [9]. The minor allele $G$ also shows lower affinity of binding forkhead box L1 (Foxl1) and homeobox D10 (Hoxd10) [10] proteins. Foxl1 encodes a member of the forkhead/winged helix-box (FOX) family of transcription factors, which play a critical role in the regulation of multiple processes including metabolism, cell proliferation and gene expression during ontogenesis.
Hoxd10 is a member of the Abd-B homeobox family and encodes a protein with a homeobox DNA-binding domain. Recent studies suggest that HoxD10 functions as a candidate tumor suppressor $[11,12]$.

A GWAS of cervical cancer (CIN2, CIN3 and cervical cancer) based on patients of mixed European descent identified associations with both $H L A-B * 07$ and $H L A-B * 15: 01$ and squamous cervical cancer (personal communication). Consistent with this, we found associations of CIN3 (all squmous cell carcinoma) with both $H L A-B * 07: 02$ and $H L A-B * 15: 01$ in our study. In addition, we confirmed associations with $H L A$ $D R B 1 * 15: 01$ and HLA-DQB1*06:02 as well as HLA-DRB1*13:01, HLA-DQA1*01:03 and HLA-

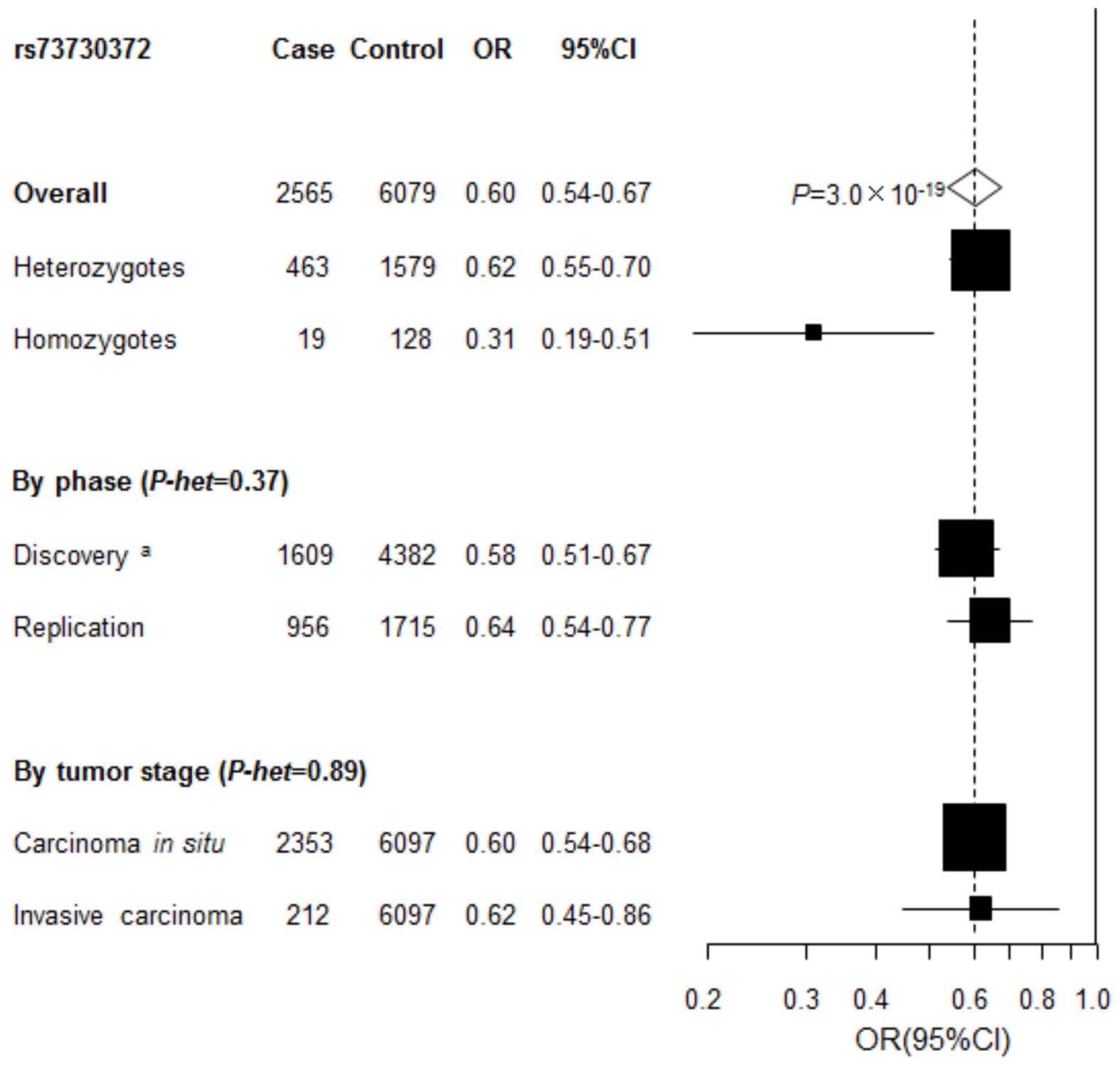

Figure 3: Association between rs73730372 and cervical disease in the combined series. Unless specified, association between rs 73730372, a proxy of $\operatorname{rs} 115625939\left(D^{\prime}=1, r^{2}=1\right)$ and cervical cancer in the combined data was estimated by logistic regression analysis for the minor allele in log-additive model with adjustment for study after pooling individual level data from the discovery and replication phase. P-het, P for heterogeneity, was derived from Cochran's $Q$ test. a Derived from logistic regression analysis for the minor allele in log-additive model with adjustment for study and the one significant principal component generated by principal components analysis. 
$D Q B 1 * 06: 03$, which are usually reported to be associated with risk of cervical cancer [13-15]. We also identified an association of CIN3 with HLA-C*07:02.

Another GWAS performed in the Chinese population reported invasive cervical carcinoma to be associated with variants in the HLA-DP region (rs4282438 and rs9277952), and with two non-MHC loci: Exocyst complex component 1 (EXOC1) at 4q12 (rs13117307) and (Gasdermin B (GSDMB) at 17q12 (rs8067378) [16]. As shown in Supplementary Table S5, none of the loci reported in this GWAS showed evidence of association in our study. The lack of success in replicating the effect of these variants could be attributed to a number of factors An important confounder is probably the different ethnic backgrounds between the Asian (Chinese) and the Caucasian (Swedish) population. The clinical endpoint also differed between these two studies, from CIN3 to cervical cancer. The differences in association seen between our study and that of the Chinese population could also be due to variability in the frequency of HPV types, intratype variation, or host-virus interactions. Since we lack information on the infecting HPV for individual cases in our study, we are unable to take this into consideration in the analyses.

Several limitations should be noted in the present study. First, our results apply to CIN3, which represents the vast majority of cases. We therefore have higher power to identify variants associated with CIN3 and a limited power to detect association with cervical cancer. Additional risk factors may be involved in progression from CIN3 to cervical cancer. However, CIN3 and cervical cancer share the same main etiological factor, namely persistent infection with oncogenic HPVs [2], indicating that the genetic susceptibility loci identified for CIN3 may have a similar effect on the two cancer stages. Second, HPV status is not available in the present study, thus our findings could relate to some behavioral characteristics associated with HPV acquisition.

In summary, by pooling data from two GWASs and imputing over 10 million genetic variants, we have been able to comprehensively examine the association of CIN3 risk with common variants, rare variants and classical HLA alleles. This study strengthened the evidence for previously postulated susceptibility loci and provided support for a novel susceptibility locus for CIN3. The present study also provides an important proof-ofprinciple that the 1000 Genomes imputation can be used to detect novel, low frequency-large effect associations, thereby extending the utility of pre-existing GWAS data.

\section{MATERIALS AND METHODS}

\section{Study population}

The SNP data of the first GWAS was generated using the Illumina Omni Express BeadChip (Illumina, San
Diego, CA) (731,422 SNPs) in the Swedish population. The details of population and QC have been described elsewhere [17]. Briefly, after stringent quality control, data from 1,034 cervical disease patients (971 with CIS and 63 with invasive carcinoma) and 3,948 control subjects were available for 632,668 SNPs, with an overall call rate of $99.92 \%$. The second GWAS data set, also from the Swedish population, was generated using the Affymetrix Genome-wide Human SNP array 5.0 with 440,794 SNP markers. After systematic QC steps, data from 616 case subjects (597 with CIN3 and 19 with cervical cancer) and 506 control subjects were available for 341,358 SNPs with an overall call rate of $99.79 \%$ [17].

Details on study design and subjects recruitment of the replication study have been described previously [18]. Briefly, the replication study consisted of 961 patients (827 with CIN3 and 123 cervical cancer) and 1,725 matched healthy controls from the Swedish population. Informed consent was obtained from all subjects, and each study was approved by the regional ethical review board. All the cases in the discovery and replication studies are squamous cell carcinoma.

\section{SNP imputation, two GWASs merging and quality control}

The details of imputation have been described previously [17]. In brief, the second GWAS data set was changed to hg19 from hg18 using the USCS LiftOver tool (http://genome.sph.umich.edu/wiki/LiftOver). Both GWAS data sets were separately phased using the shapeittool (https://mathgen.stats.ox.ac.uk/genetics_software/ shapeit/shapeit.html) (19). They were then separately split into chunks of approximate $5 \mathrm{Mb}$ containing at least 200 genotyped SNPs. No chunks spanned the centromeres. Genetic variants across each chunk not genotyped on the Ilumina HumanOmniExpress BeadChip or Affymetrix Genome-wide Human SNP arrays 5.0 were imputed, respectively, using the program IMPUTE2 [20] with phased haplotypes in subjects from the December 2013 release of the 1000 Genomes Project data (https:// mathgen.stats.ox.ac.uk/impute/impute_v2.html\#reference) [21] as a scaffold. Imputed variants with info-score $<0.3$ in either data set were removed. The two data sets were then merged and markers with less than 0.9 in posterior genotypic probability in more than $5 \%$ of the samples or monomorphic were removed. Finally, genotypes were called from posterior probabilities using 0.9 as threshold using gtool (http://www.well.ox.ac.uk/ cfreeman/ software/gwas/gtool.html). Further QC was applied to the merged data set. The exclusion criteria for genetic variants were: (1) genotype frequency that deviated from Hardy-Weinberg Equilibrium (HWE) among control subjects $\left(P<1 \times 10^{-7}\right)$, and (2) a statistically significant difference in minor allele frequency (MAF) between the two GWASs $\left(P<1 \times 10^{-7}\right)$. As shown in Supplementary 
Table S6, unexpected duplicates (11 subjects) and firstdegree and second-degree relatives (9 subjects) were removed based on identity-by-state (IBS) estimates calculated in PLINK [22]. Utilizing a set of 15,872 SNPs genotyped in both GWASs and evenly distributed across the genome in low linkage disequilibrium (LD) (pair-wise $r^{2}<0.02$ ), a principal components analysis (PCA) using the EIGENSTRAT software [23] excluded 8 additional samples detected as outliers.

\section{Genotyping and quality control}

A genotyping assay for rs115625939 was not possible to design. To validate the findings from the whole-genome scan, SNP rs73730372 was genotyped for replication as a proxy for $\operatorname{rs} 115625939\left(D^{\prime}=1, r^{2}=1.00\right)$ with template-directed dye-terminator incorporation with fluorescence-polarization detection (FP-TDI) (Tecan, Männedorf, Switzerland). SNP rs73000875 was also genotyped using the same assay. Eight percent of the samples were selected for repeat genotyping as duplicates, yielding a reproducibility of $100 \%$. Genotype success rate was greater than $98.7 \%$ and genotype distributions were consistent with that expected by HWE for each SNP.

\section{Statistical analysis}

In the discovery phase, the potential for population stratification was investigated by PCA undertaken with the EIGENSTRAT package [23]. One significant PC derived from PCA based on the Tracy-Widom statistic $(P<0.05)$ was statistically significantly associated with case-control status $(P<0.05)$. Adjustment for population stratification was performed by including significant PC as covariate in the logistic regression. The association between each genetic variant and risk of cervical disease was estimated by the OR per minor allele and 95\% CI using multivariate unconditional logistic regression, assuming a log-additive model with adjustment for study and the significant PC with PLINK. Conditioning analysis on significant HLA alleles/haplotypes was performed in PLINK using logistic regression in logadditive model conditioning on $B * 07: 02-C * 07: 02, B * 15: 01$, $D R B 1 * 13: 01-D Q A 1 * 01: 03-D Q B 1 * 06: 03$ and $D R B 1 * 15: 01-$ $D Q B 1 * 06: 02$ with adjustment for study and one significant principal component generated by principal components analysis. The conditioning HLA alleles/haplotypes were entered into the model simply as covariates.

In the replication study, the association between each genetic variant and risk of cervical disease was estimated by the OR per minor allele and 95\% CI using unconditional logistic regression assuming a logadditive model. Individual level data from the discovery and replication phase were pooled, making for a total of 2,565 cervical cases and 6,079 controls. In the combined analysis, association between each genetic variant and risk of cervical disease was estimated by the OR per minor allele and 95\% CI using unconditional logistic regression assuming a log-additive model with adjustment for study. We then conducted stratified analysis by study phase and tumor grade. Heterogeneity of ORs was assessed using the Cochran's Q test. Results that obtained a level of significance of a $P<5.0 \times 10^{-8}$ were considered statistically significant at the genomewide level. All replication and combined analyses were conducted using SAS 9.3 software. All statistical tests were two-sided.

\section{Imputation at classical HLA alleles and analysis}

The T1DGC panel contains 5,868 SNPs (genotyped with Illumina Immunochip) and 4-digit classical HLA types for $H L A-A,-B,-C,-D P B 1,-D Q A 1$, $-D Q B 1$ and $-D R B 1$ for 5,225 unrelated individuals (10,450 haplotypes) [24]. With the T1DGC reference panel, we imputed 126 classical 2-digit alleles and 298 classical 4-digit alleles at $H L A-A,-B,-C,-D P B 1$, $-D Q A 1,-D Q B 1$ and $-D R B 1$ using SNP2HLA [24]. Unconditional logistic regression using the imputed genotype for each classical HLA allele was carried out using PLINK with adjustment for study and the significant PC identified from PCA.

\section{Genotyping of classical HLA loci}

The typing of $H L A-A,-B,-D R B 1,-D Q B 1$ and $-D P B 1$ in 254 cervical cancer patients included in the present study was previously performed [25] by PCR amplification of groups of alleles using biotinylated PCR primers, followed by hybridization to immobilized sequence-specific oligonucleotide probes in a lineararray format. Genotypes were determined using a computer algorithm on the basis of the pattern of sequence-specific oligonucleotide-probe hybridization.

\section{ACKNOWLEDGMENTS AND FUNDING}

This work was supported by the National Natural Science Foundation of China (NSFC) grants (81401212, 91331204; 31501011), by the Youth Eastern Scholar (QD 2015006), by the Pujiang Talent Project (15PJ1405500), by the Strategic Priority Research Program of the Chinese Academy of Sciences (CAS) (XDB13040100), by the National Science Fund for Distinguished Young Scholars (31525014), by the Program of Shanghai Academic Research Leader (16XD1404700) S.X. also gratefully acknowledges the support of the National Program for Top-notch Young Innovative Talents of the "Wanren Jihua" Project.

\section{CONFLICTS OF INTEREST}

None declared. 


\section{REFERENCES}

1. Ferlay J, Soerjomataram I, Dikshit R, Eser S, Mathers C, Rebelo M, Parkin DM, Forman D, Bray F. Cancer incidence and mortality worldwide: sources, methods and major patterns in GLOBOCAN 2012. Int J Cancer. 2015; 136:E359-386.

2. Schiffman M, Wentzensen N. Human papillomavirus infection and the multistage carcinogenesis of cervical cancer. Cancer Epidemiol. Biomarkers Prev. 2013; 22:553-560.

3. Chen D, Juko-Pecirep I, Hammer J. Genome-wide association study of susceptibility loci for cervical cancer. J Natl Cancer Inst. 2013; 105: 624-633.

4. GTEx Consortium. Human genomics. The GenotypeTissue Expression (GTEx) pilot analysis: multitissue gene regulation in humans. Science. 2015; 348:648-660.

5. Stanley M. Immune responses to human papillomavirus. Vaccine. 2006; 24:S16-22.

6. Woodworth CD, Simpson S. Comparative lymphokine secretion by cultured normal human cervical keratinocytes, papillomavirus-immortalized, and carcinoma cell lines. Am J Pathol. 1993; 142:1544-1555.

7. Coleman N, Birley HD, Renton AM. Immunological events in regressing genital warts. Am J Clin Pathol. 1994; 102:768-774.

8. Arany I, Tyring SK. Activation of local cell-mediated immunity in interferon-responsive patients with human papillomavirus associated lesions. J Interferon Cytokine Res. 1996; 16:453-460.

9. Stanley MA. Immune responses to human papilloma virus. Indian J Med Res. 2009; 130:266-276.

10. Kheradpour P, Kellis, M. Systematic discovery and characterization of regulatory motifs in ENCODE TF binding experiments. Nucleic Acids Res. 2014; 42:2976-2987.

11. Wang L, Chen S, Xue M. Homeobox D10 gene, a candidate tumor suppressor, is downregulated through promoter hypermethylation and associated with gastric carcinogenesis. Mol Med. 2012; 18:389-400.

12. Nakayama I, Shibazaki M, Yashima-Abo A. Loss of HOXD10 expression induced by upregulation of miR-10b accelerates the migration and invasion activities of ovarian cancer cells. Int J Oncol. 2013; 43:63-71.

13. Zoodsma M, Nolte IM T, Meerman GJ. HLA genes and other candidate genes involved in susceptibility for (pre) neoplastic cervical disease. Int J Oncol. 2005; 26:769-784.
14. Hildesheim A, Wang SS. Host and viral genetics and risk of cervical cancer: a review. Virus Res. 2002; 89:229-240.

15. Krul EJT, Schipper RF, Schreuder GMT, Fleuren GJ, Kenter GG, Melief CJM. HLA and susceptibility to cervical neoplasia. Hum Immunol. 1999; 60:337-342.

16. Shi Y, Li L, Hu Z. A genome-wide association study identifies two new cervical cancer susceptibility loci at 4q12 and 17q12. Nat Genet. 2013; 45:918-922.

17. Chen D, Enroth S, Ivansson E. Pathway analysis of cervical cancer genome-wide association study highlights the MHC region and pathways involved in response to infection. Hum Mol Genet. 2014; 23:6047-6060.

18. Chen D, Hammer J, Lindquist D. A variant upstream of HLA-DRB1 and multiple variants in MICA influence susceptibility to cervical cancer in a Swedish population. Cancer Med. 2014; 3:190-198.

19. Delaneau O, Zagury JF, Marchini J. Improved whole chromosome phasing for disease and population genetic studies. Nat Methods. 2013; 10:5-6.

20. Howie BN, Donnelly P, Marchini J. A flexible and accurate genotype imputation method for the next generation of genome-wide association studies. PLoS. Genetics. 2009; 5: e1000529.

21. 1000 Genomes Project Consortium, Abecasis GR, Altshuler D. A map of human genome variation from population-scale sequencing. Nature. 2010; 467:1061-1073.

22. Purcell S, Neale B, Todd-Brown K. PLINK: a tool set for whole-genome association and population based linkage analyses. Am J Hum Genet. 2007; 81:559-575.

23. Price AL, Patterson NJ, Plenge RM. Principal components analysis corrects for stratification in genome-wide association studies. Nat Genet. 2006; 38:904-909.

24. Jia X, Han B, Onengut-Gumuscu S. Imputing amino acid polymorphisms in human leukocyte antigens. PLoS One. 2013; 8:e64683.

25. Engelmark M, Beskow A, Magnusson J. Affected sib-pair analysis of the contribution of HLA class I and class II loci to development of cervical cancer. Hum Mol Genet. 2004; 13:1951-1958. 